\title{
Features of subsyndromal and persistent delirium
}

\author{
David Meagher, Dimitrios Adamis, Paula Trzepacz and Maeve Leonard
}

\author{
Background \\ Longitudinal studies of delirium phenomenology are \\ lacking.
}

\begin{abstract}
Aims
We studied features that characterise subsyndromal delirium and persistent delirium over time.

\section{Method}

Twice-weekly evaluations of 100 adults with DSM-IV delirium using the Delirium Rating Scale - Revised-98 (DRS-R98) and Cognitive Test for Delirium (CTD). The generalised estimating equation method identified symptom patterns distinguishing full syndromal from subsyndromal delirium and resolving from persistent delirium.
\end{abstract}

\section{Results}

Participants (mean age 70.2 years $($ s.d. $=10.5)$ ) underwent 323 assessments (range 2-9). Full syndromal delirium was significantly more severe than subsyndromal delirium for
DRS-R98 thought process abnormalities, delusions, hallucinations, agitation, retardation, orientation, attention, and short- and long-term memory items, and CTD attention, vigilance, orientation and memory. Persistent full syndromal delirium had greater disturbance of DRS-R98 thought process abnormalities, delusions, agitation, orientation, attention, and short- and long-term memory items, and CTD attention, vigilance and orientation.

\section{Conclusions}

Full syndromal delirium differs from subsyndromal delirium over time by greater severity of many cognitive and noncognitive symptoms. Persistent delirium involves increasing prominence of recognised core diagnostic features and cognitive impairment.

\section{Declaration of interest}

P.T. is a full-time salaried employee and shareholder at Eli Lilly and Company.
Delirium is a complex neuropsychiatric syndrome occurring in $11-42 \%$ of general medical in-patients, ${ }^{1}$ with even higher rates in older patients, those with pre-existing cognitive impairment, terminal illness or receiving care in intensive care units. ${ }^{2}$ Although delirium is classically described as a syndrome with high inherent reversibility, studies have increasingly highlighted that persistence is a common occurrence in older people. ${ }^{3}$ Despite its relatively high incidence and potential for a range of negative outcomes including higher mortality and reduced independent functioning in elderly people, delirium remains seriously understudied. ${ }^{4}$ Recent cross-sectional studies that utilise more detailed measurement methods provide a valuable insight into the symptoms that occur most frequently and at greater severity in patient groups with delirium. ${ }^{5-8}$ However, longitudinal studies that focus on describing severity, temporal course or symptom pattern of delirium episodes are relatively lacking; Fann et al ${ }^{9}$ used the Delirium Rating Scale (DRS) and Memorial Delirium Assessment Scale (MDAS) items together for daily ratings, Sylvestre et $a l^{10}$ used the Delirium Index to describe overall patterns of severity and Rudberg et al ${ }^{11}$ reported daily scores on the DRS in surgical patients. However, only Fann et al reported individual symptoms over time. Therefore, the pattern, interrelationships and temporal course of symptoms during episodes are still not well understood.

Elucidating subsyndromal delirium symptoms could serve to enhance early recognition of individuals at risk for delirium or poorer outcomes. Identification of delirium symptoms that occur in individuals who do not meet full diagnostic criteria either as a prodrome, milder state or transient recovery phase has not yet revealed specific symptoms that could consistently define a state of subsyndromal delirium. ${ }^{7}$ Subsyndromal delirium, as variously defined, appears to carry prognostic significance for adverse outcomes that are intermediate between full syndromal delirium and controls (for example, decreased post-discharge level of functional independence, elevated mortality, increased length of stay). ${ }^{12-16}$ However, DSM-IV ${ }^{17}$ criteria are known to be the least restrictive to diagnose delirium ${ }^{18}$ as compared with ICD- $10^{19}$ and DSM-III- $\mathrm{R}^{20}$ and therefore likely to incorporate individuals with milder and subsyndromal delirium under its umbrella.

We report a prospective longitudinal assessment of phenomenological profile in 100 consecutive participants with DSM-IV-defined delirium examining (a) the pattern of symptoms over time in both full syndromal and subsyndromal delirium, and (b) symptom pattern in individuals with delirium with resolving v. persisting delirium.

\section{Method}

\section{Participants and design}

All patients admitted to a palliative care unit in Limerick, Ireland (Milford Care Hospice) with cancer diagnoses were assessed daily for altered mental states and where appropriate formally screened with the Confusion Assessment Method (CAM) algorithm ${ }^{21}$ by the medical team who are trained in using the CAM in order to supplement routine case-finding for delirium. Training procedures were developed as part of a validation study of the CAM in palliative care settings. ${ }^{22}$ Individuals with CAM-positive status or other altered mental states are referred to the liaison psychiatry service for further evaluation and expert diagnosis of delirium. In order to optimise the real-world nature of this study, both incident and prevalent cases of delirium were included.

Individuals meeting DSM-IV criteria for delirium ${ }^{17}$ underwent a detailed assessment of demographics, phenomenological profile, dementia status and longitudinal course of delirium. Assessments were conducted by trained raters (M.L. or D.M.) with established high interrater reliability, experience and expertise in administration of the Delirium Rating Scale - Revised-98 (DRS-R98) ${ }^{23}$ and 
the Cognitive Test for Delirium (CTD). ${ }^{24}$ To further enhance interrater reliability, difficult ratings were discussed and rated by consensus between both raters. The DRS-R98 was followed by administration of the CTD to keep the cognitive elements between the scales separate. The DRS-R98 rated the preceding 72-hour period, whereas the CTD measured cognition at the time of its administration. The CTD responses were not used to rate DRS-R98 items.

For this serial assessment study, assessments were repeated twice weekly for 3 weeks, and weekly thereafter, until either the episode of delirium resolved, patients were discharged or their medical condition seriously deteriorated such that it became appropriate to cease involvement in the study (as agreed with the treating medical team and/or family carers), which resulted in a maximum of nine study visits. Study participation was discontinued at any stage if either the participant or family indicated a desire to exit the study. Although 121 consecutive participants with DSM-IV delirium were assessed, only 100 individuals underwent at least two assessments and are included in this serial analysis study.

Although all participants met DSM-IV criteria for delirium at study entry, for the purposes of analysis DRS-R98 cut-off scores were used to identify delirium syndromal status and compared over time with regard to (a) full syndromal delirium $v$. subsyndromal delirium, and (b) those that followed a resolving (initially full syndromal delirium becoming subsyndromal delirium over time) $v$. persisting delirium course (maintaining full syndromal delirium throughout all assessments).

\section{Procedures}

Delirium Rating Scale - Revised-98

The DRS-R98 ${ }^{23}$ is a widely used instrument, with multiple language translations and revalidations, that measures symptom profile in delirium and is validated as both a diagnostic and severity assessment tool. It is a 16 -item clinician-rated scale with 13 severity items and 3 diagnostic items. It is sensitive to change over time and is frequently used in drug treatment studies as it is suited to longitudinal studies. The 13-item severity section can be scored separately from the 3-item diagnostic section; their sum constitutes the total scale score with a maximum score of 46 . All items are anchored by text descriptions that guide rating along a continuum from normal (0) to severely impaired (3) for severity items and from 0 to either 2 or 3 for diagnostic items. The DRS-R98 severity scale score ranges from 0 to 39 with higher scores indicating more severe delirium and a cut-off score $\geqslant 15$ is consistent with a diagnosis of delirium. Severity scale scores between 8 and 15 are considered subsyndromal in severity (subsyndromal delirium). ${ }^{25}$

Assessments take approximately $10-15 \mathrm{~min}$ and are made according to all available sources of information, including assessment of the patient and discussion with nursing staff and family/carers. The DRS-R98 is designed to rate symptoms over the previous $24 \mathrm{~h}$ but can also be used to rate symptoms over variable periods from hours to weeks and for the purposes of this study was applied twice weekly to encompass the previous 3-4 day period (i.e. since last assessment). It has high interrater reliability, validity, sensitivity and specificity for distinguishing delirium from mixed neuropsychiatric populations including dementia, depression and schizophrenia. ${ }^{23}$

\section{Cognitive Test for Delirium}

The $\mathrm{CTD}^{24}$ is designed to assess neuropsychological function in medically ill individuals with delirium. It takes approximately
15-20 min to administer and emphasises non-verbal (visual and auditory) over verbal abilities. It is thus suitable for use in a broad range of individuals including those who are intubated or otherwise cannot speak. It was originally designed for use in severely ill people in intensive care units but has been used to study delirium in other medical in-patient groups ${ }^{6,23}$ including individuals with traumatic brain injury. ${ }^{26}$ It measures five neuropsychological domains (orientation, attention, memory, comprehension and vigilance). It generates a score of between 0 and 30, with higher scores indicating better cognitive function. An optimal cut-off score to discriminate delirium from other disorders is $<19$, however it can be used as a continuous, unidimensional measure of cognition in delirium. ${ }^{26}$ It reliably distinguishes between dementia, schizophrenia and depression. ${ }^{27}$

\section{Pre-existing cognitive impairment and dementia}

Dementia was defined as the presence of persistent cognitive impairment for at least 6 months prior to the assessment and per DSM criteria based on all available information at the time of initial assessment including clinical case notes and collateral history from family and/or carers.

\section{Medication exposure}

All medications prescribed for the participant were documented at each visit including regular and as-required doses. The use of psychoactive agents was a specific focus, especially the use of antipsychotic, opioid, benzodiazepine, psychostimulant or corticosteroid agents that have been reported to be associated with delirium as a risk factor/aetiology or treatment. The number of medications in these classes were summed for each visit and dose equivalents over the previous $24 \mathrm{~h}$ were calculated for each of these drug classes according to accepted conversion rates (i.e. antipsychotics in chlorpromazine equivalents; opioids in morphine equivalents; benzodiazepines in diazepam equivalents; steroids in prednisolone equivalents). ${ }^{28-30}$

\section{Informed consent}

The procedures and rationale for the study were explained to all participants but because the majority of participants included in this work were identified as having an index episode of delirium at entry into the study, it was presumed that most would not be capable of giving informed written consent. Because of the noninvasive nature of the study, the Limerick Regional Ethics Committee approved an approach whereby patient verbal assent was augmented with proxy consent from next of kin (where possible) or a responsible caregiver. This is in accordance with best practices as outlined in the Helsinki guidelines for medical research involving human subjects. ${ }^{31}$ A standard information sheet was provided for all components of the study. This study did not have any identified significant risks and participants and/or family were informed that they could withdraw participation at any time.

\section{Statistical analysis}

Demographic data and baseline characteristics are listed using descriptive methods including mean and standard deviation. Little's MCAR (missing completely at random) test indicated that there was no systematic pattern of missing values $\left(\chi^{2}=119.5\right.$; d.f. $=219, P=1.0)$. Stata 10 and S-PLUS 6 software for Windows $\mathrm{XP}$ were used.

The generalised estimating equations method (GEE) was used to analyse longitudinal data for patterns of individual items from the 
DRS-R98 and CTD within and between defined groups for full syndromal delirium/subsyndromal delirium and resolving/persistent delirium over the study period. The group designation at baseline and at each subsequent assessment (full syndromal delirium or subsyndromal delirium) was determined according to the predefined DRS-R98 score cut-off point. The GEE approach was used to find pattern changes across the visits. The GEE method takes into account the fact that observations within a participant are correlated and estimates the population average across time. The estimated coefficients reflect the relationship between the longitudinal development of the dependent variable (grouped by delirium status full syndromal delirium $v$. subsyndromal delirium) and the longitudinal development of the predictor variables (for example, age, gender, DRS-R98 items) using all data. Specifically, the coefficient represents the change in logit of the outcome variable (i.e. full syndromal delirium $v$. subsyndromal delirium) associated with a one-unit change in the predictor variable, where the logit is the natural logarithm of the odds of the outcome occurring. All scale items were analysed in each analysis, although only those that were significantly different are shown in the results tables, with the reference group listed first. Scoring for impairment on the DRS-R98 is opposite to that of the CTD.

Although all participants met DSM-IV criteria for delirium at study entry, not all met the DRS-R98 total scale cut-off value $(\geqslant 16)$ for full syndromal delirium. Participants were divided according to a resolving (i.e. full syndromal delirium becoming subsyndromal delirium over time) or persisting delirium course (i.e. maintaining full syndromal delirium over time) according to the evolution of DRS-R98 scores, and again assessed for symptom evolution using the GEE approach. To produce a visual representation of the DRS-R98 items over time, smoothing techniques and trellis graphs were used.

\section{Results}

\section{Sample description}

The 100 participants underwent a total of 323 observations for up to nine visits over 6 weeks (twice weekly for 3 weeks, followed by weekly thereafter), with fewer participants remaining in the study population as time went on (see Fig. 1 for numbers evaluated at each visit). Although at the first assessment all participants met DSM-IV criteria for delirium, according to DRS-R98 scores, 73 participants were categorised with full syndromal delirium whereas 27 had subsyndromal delirium. Overall, of the 323

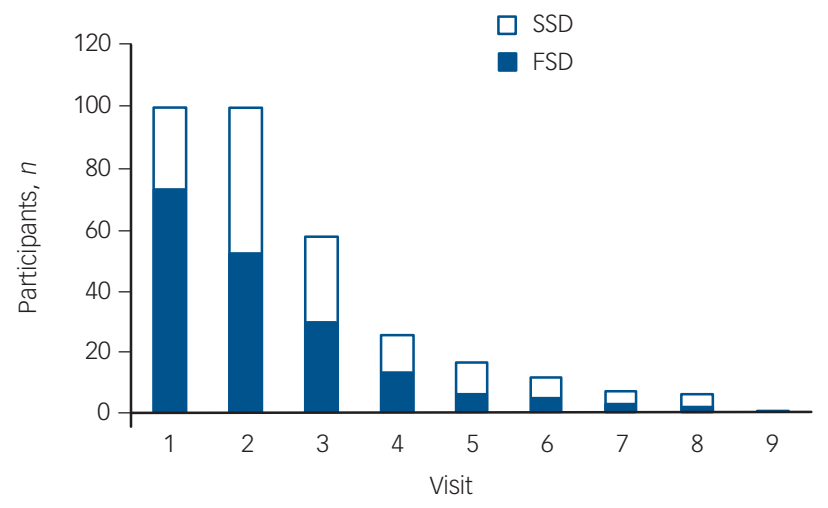

Fig. 1 Frequency of participants with full syndromal and subsyndromal delirium at each study visit. Visits 1-6 occurred twice weekly and visits 7-9 were weekly. SSD, subsyndromal delirium; FSD, full syndromal delirium.

\begin{tabular}{|c|c|}
\hline & $\begin{array}{l}\text { Study population } \\
\qquad(n=100)\end{array}$ \\
\hline Age, years: mean (s.d.) range & 70.2 (10.5) 36-90 \\
\hline Gender, male/female: \% & $51 / 49$ \\
\hline Comorbid dementia, \% & 27 \\
\hline $\begin{array}{l}\text { Number of aetiologies per participant, } \\
\text { mean (s.d.) range }\end{array}$ & $3.4(1.2) 1-7$ \\
\hline $\begin{array}{l}\text { Time from first assessment to death, days: } \\
\text { mean (s.d.) range }\end{array}$ & 45.3 (54.7) 3-320 \\
\hline $\begin{array}{l}\text { Delirium Rating Scale - Revised-98, mean (s.d.) range } \\
\text { Severity } \\
\text { Total }\end{array}$ & $\begin{array}{l}16.2(6.3) 5-36 \\
20.4(6.3) 8-40\end{array}$ \\
\hline Cognitive Test for Delirium, total: mean (s.d.) range & $14.2(7.7) 0-30$ \\
\hline
\end{tabular}

assessments, 190 (58\%) met full syndromal delirium cut-off scores according to DRS-R98.

Clinical and demographic characteristics are shown in Table 1. Reasons for discontinuation from the study were death $(n=55)$, recovery $(n=30)$, declined further participation $(n=12)$ and discharged $(n=3)$. Medication use was considerable such that all participants were receiving medications (mean number of medications 10.3 per participant (s.d.=3.2) over all assessments; range 2-20). Use of psychoactive medications over the course of episodes was $87 \%$ receiving opioids, $76 \%$ receiving antipsychotics, $72 \%$ receiving benzodiazepines, $54 \%$ receiving corticosteroids and $1 \%$ receiving psychostimulants.

\section{Full syndromal delirium v. subsyndromal delirium analyses}

We used the GEE method to identify symptoms with a significant effect on delirium status over time. Delirium status (full syndromal $v$. subsyndromal delirium) was the binary dependent variable, with age, gender, 13 DRS-R98 severity items and dose equivalents of psychoactive agent classes used as the independent variables.

The final most parsimonious GEE model shows the DRS-R98 items significantly associated with full syndromal rather than subsyndromal delirium over time in Table 2. Delusions, hallucinations, thought process disturbance, motor retardation, motor agitation, orientation, attention, and short- and long-term memory were all significantly higher (more impaired) in full syndromal $v$. subsyndromal delirium, whereas other independent variables including medication exposure were not significantly associated with delirium syndromal status.

We performed a similar analysis for the CTD to compare full syndromal with subsyndromal delirium. The dependent variable was full syndromal delirium (as a dichotomous variable using DRS-R98 cut-off score of 16) and the independent variables were the five cognitive domains measured with CTD plus demographic variables (age and gender) and medication status. The final most parsimonious model is presented in Table 3. Lower scores (greater impairment) on all CTD items except comprehension and higher benzodiazepine doses were significantly associated with full syndromal status over time.

In subsequent analyses we also included comorbid dementia as a variable but this did not significantly impact on the findings for the DRS-R98 or CTD items and as such the models for the complete cohort are shown.

Figure 2 is the representation produced by Friedman's Smoothing method of the 13 individual DRS-R98 items over time 
Table 2 Generalised equation estimation model findings for Delirium Rating Scale - Revised-98 (DRS-R98) severity item scores for full syndromal delirium as compared with subsyndromal delirium ${ }^{\mathrm{a}}$

\begin{tabular}{|lcccccc|}
\hline DRS-R98 & Coefficient & S.e. & $P$ & Wald $\chi^{2}$ & d.f. & $95 \% \mathrm{Cl}$ \\
\hline Thought process abnormalities & 0.90 & 0.43 & 0.038 & 4.357 & 1 & 0.51 to 1.75 \\
\hline Delusions & 3.20 & 1.00 & 0.001 & 10.383 & 1 & 1.25 to 5.17 \\
\hline Hallucinations & 1.45 & 0.41 & 0.001 & 12.463 & 1 & 0.64 to 2.25 \\
\hline Agitation & 2.68 & 0.59 & 0.001 & 20.379 & 1 & 1.51 to 3.85 \\
\hline Retardation & 2.90 & 0.67 & 0.001 & 19.067 & 1 & 1.59 to 4.22 \\
\hline Orientation & 1.52 & 0.57 & 0.009 & 6.962 & 1 & 0.38 to 2.65 \\
\hline Attention & 1.77 & 0.45 & 0.001 & 15.584 & 1 & 0.89 to 2.66 \\
\hline Short-term memory & 1.57 & 0.34 & 0.001 & 21.389 & 1 & 0.91 to 2.25 \\
\hline Long-term memory & 1.75 & 0.55 & 0.002 & 9.964 & 1 & 0.66 to 2.84 \\
\hline Constant & -13.35 & 2.57 & 0.001 & 27.214 & 1 & -18.40 to -8.31 \\
\hline a. Only significant values are shown. Higher DRS-R98 scores are more impaired. & & & & \\
\hline
\end{tabular}

Table 3 Generalised equation estimation model findings for Cognitive Test for Delirium (CTD) items for full syndromal delirium as compared with subsyndromal delirium ${ }^{\mathrm{a}}$

\begin{tabular}{|lcccccc|}
\hline CTD item & Coefficient & s.e. & Wald $\chi^{2}$ & d.f. & $P$ & $95 \% \mathrm{Cl}$ \\
\hline Attention & -0.39 & 0.11 & 13.4 & 1 & $<0.001$ & -0.60 to -0.18 \\
\hline Memory & -0.29 & 0.09 & 10.3 & 1 & 0.001 & -0.47 to -0.11 \\
\hline Vigilance & -0.22 & 0.10 & 4.4 & 1 & 0.03 & -0.42 to -0.02 \\
\hline Orientation & -0.26 & 0.10 & 7.2 & 1 & 0.007 & -0.46 to -0.07 \\
\hline Diazepam dose equivalents & 0.07 & 0.1 & 6.6 & 1 & 0.01 & 0.02 to 0.14 \\
\hline Constant & 4.18 & 0.58 & 51.8 & 1 & $<0.001$ & 3.04 to 5.32 \\
a. Only significant items are shown. Lower CTD scores are more impaired. & & & & \\
\hline
\end{tabular}

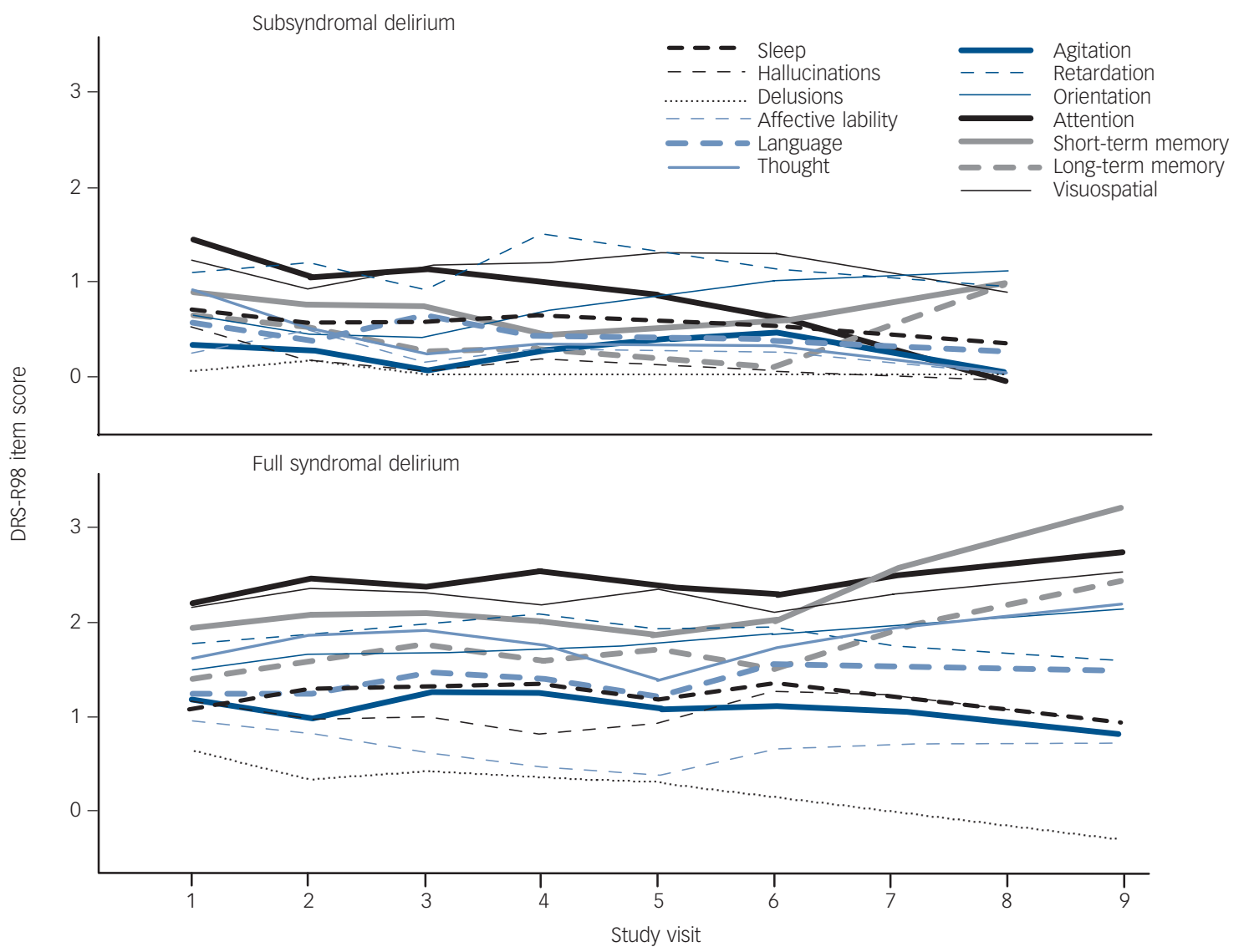

Fig. 2 Comparison of Delirium Rating Scale - Revised-98 (DRS-R98) item scores over time (up to nine visits) in subsyndromal delirium (top) and full syndromal delirium (bottom). 
graphed for full syndromal delirium and subsyndromal delirium groups. These are scatter plots overlaid by smoothed linear regression lines. Similarly, Fig. 3 shows the five CTD items over time graphed for full syndromal and subsyndromal delirium groups. In both subsyndromal delirium and full syndromal delirium, symptom evolution did not follow an entirely unitary pattern but, in general, symptom severities were relatively stable across assessments with a pattern of increasing cognitive impairment towards the later assessments in the full syndromal delirium category

\section{Resolving $\boldsymbol{v}$. persisting delirium}

In order to identify symptoms that are most relevant to persisting delirium we performed GEE analyses comparing symptom profile over time in resolving cases (initially meeting full syndromal delirium criteria but now subsyndromal delirium) $v$. persisting full syndromal delirium. Twenty-seven participants with subsyndromal delirium at study entry were excluded from this analysis. We performed a GEE using DRS-R98 cut-off of 16 to distinguish resolving from persistent delirium and entered the DRS-R98 individual items as independent variables. The final parsimonious model is shown in Table 4. Items that best predicted the likelihood of persisting delirium were more severe disturbances of orientation, short- and long-term memory, delusions, motor agitation, inattention and thought process abnormalities.

A similar analysis was performed using the CTD items as independent variables and the final model is shown in Table 5. Greater impairment (lower scores) for attention, vigilance and orientation best distinguished persisting from resolving delirium. In addition, persistent delirium was associated with greater benzodiazepine exposure over time.

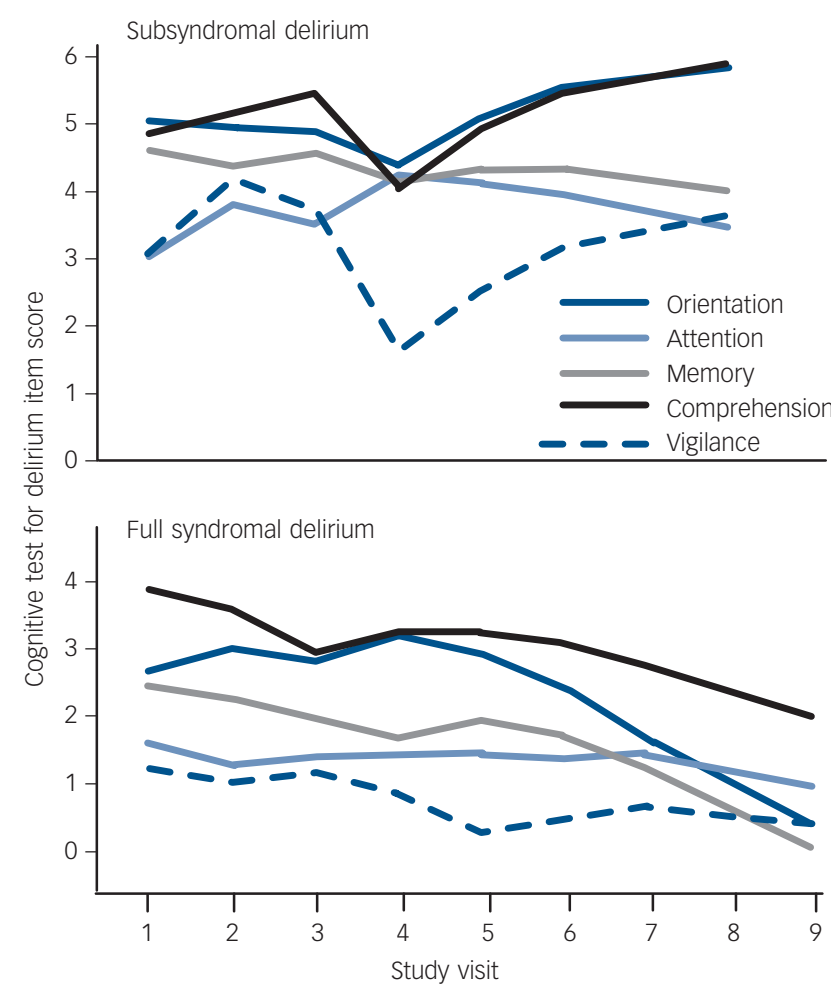

Fig. 3 Comparison of Cognitive Test for Delirium (CTD) item scores over time (up to nine visits) in full syndromal and subsyndromal delirium.
These analyses were repeated with comorbid dementia as a predictor variable, but again the findings were not substantially altered.

\section{Discussion}

\section{Main findings}

This study is the first to describe the longitudinal pattern and severity of individual delirium symptoms utilising the GEE method. We also assessed how different elements of this complex neuropsychiatric syndrome are relevant to delirium full syndromal status and persistence. This method explores patterns according to population averages rather than for individual patients and is therefore suited to evaluating particular phenomenological states that vary over the course of an episode of delirium. We captured symptoms using two well-validated and specific scales for quantifying delirium phenomenology, the DRS-R98 and the CTD. We also controlled for several variables, including exposure to psychoactive medications.

Delirium is a syndrome with a defined set of characteristic symptoms, many of which occur commonly though not in every individual at the same frequency or severity. Although much more is known about how these symptoms are expressed crosssectionally, little is known about their temporal pattern during an episode. We found that all delirium symptoms continue throughout an episode until its resolution and also occur in subsyndromal delirium, although some to a milder degree, confirming that delirium is indeed a cohesive syndrome. Most symptoms had only modest fluctuations across time, although there was a change at the end of more prolonged episodes for a small number of symptoms (for example, inattention, thought process abnormality). Symptoms that are more consistently present over the course of illness may hold special relevance to improving delirium detection and monitoring progress over time.

Longitudinal studies to date have been hampered by methodological issues with inadequate assessment tools to assess the broad range of symptoms that occur in delirium. Fann et al ${ }^{9}$ studied delirium symptoms over an 8 -week period and found that non-cognitive features dominated in the prodromal phase with cognitive symptoms peaking at 1 week. Our work also identifies increasing dominance of cognitive symptoms over time with increasing prominence of orientation, short- and long-term memory, motor agitation, delusions, disorganised thinking and disturbances of attention on the DRS-R98.

Overall, inattention and disorganised thinking were the most significant DRS-R98 features that distinguished more persistent delirium. Similarly, for the CTD the tests of attention and vigilance were the most distinguishing. Our findings emphasise disturbance of attention and disturbed thinking as dominant elements throughout the course of delirium and are consistent with cross-sectional work that highlights inattention as a core feature of delirium. ${ }^{6}$ Although initial entry to the study was according to DSM-IV criteria, which require inattention, it is relevant that impaired attention on both the DRS-R98 and CTD continues to be a key feature during persistent full syndromal delirium.

\section{Use of the GEE modelling method}

Given the challenges of handling the effects of individuals dropping out, interdependence of ratings across visits within participants, and individual participant's variability in delirium severity over time, we chose the GEE modelling method because it manages these issues in longitudinal data-sets. This method has the advantage of exploring patterns according to population 


$\begin{aligned} & \text { Table } 4 \\ & \text { as compared with resolving delirium }(n=73)\end{aligned}$
$\begin{aligned} & \text { DRS-R98 items } \\ & \text { Coefficient }\end{aligned}$

\begin{tabular}{|c|c|c|c|c|c|c|}
\hline CTD item & Coefficient & s.e. & Wald $\chi^{2}$ & d.f. & $P$ & $95 \% \mathrm{Cl}$ \\
\hline Attention & -0.55 & 0.12 & 8.92 & 1 & $<0.001$ & -0.78 to -0.32 \\
\hline Vigilance & -0.34 & 0.11 & 9.39 & 1 & 0.002 & -0.56 to -0.12 \\
\hline Orientation & -0.27 & 0.09 & 8.67 & 1 & 0.003 & -0.46 to -0.09 \\
\hline Diazepam dose equivalents & 0.11 & 0.04 & 8.91 & 1 & 0.003 & 0.04 to 0.18 \\
\hline Constant & 4.32 & 0.56 & 58.47 & 1 & $<0.001$ & 3.21 to 5.43 \\
\hline
\end{tabular}

averages rather than for individual patients and is therefore suited to evaluating particular phenomenological states that vary over the course of an episode of delirium such that individuals can change category. To date in delirium research, a small number of other studies have used the GEE modelling method, although not applied to phenomenology. Dolan et al ${ }^{32}$ used GEE to examine the prognostic significance of delirium on 2-year functional outcomes. Gaudreau et $a^{33}$ used GEE to examine the relationship between corticosteroid, benzodiazepine and opioid use on the longitudinal risk of delirium. Cheung $e t a l^{34}$ surveyed the recognition of delirium by Canadian intensivists using GEE and found that the presence of an obvious medical aetiology was a key factor in accurate identification of delirium.

\section{Subsyndromal delirium}

Although all participants met DSM-IV criteria for delirium at baseline, $27 \%$ were considered to have subsyndromal delirium according to DRS-R98 cut-off scores. Subsyndromal delirium was first described by Lipowski ${ }^{35}$ and has subsequently been defined by either the presence of any core symptoms without full diagnostic criteria or severity scores on rating scales that are below the diagnostic threshold. No uniform definition is available yet, however, and there is a paucity of research. Subsyndromal presentations may be common, although depend on definitional clarification $^{36}$ and are associated with outcomes that are intermediate between those with and without delirium in terms of morbidity, mortality and length of hospital stay. ${ }^{13,14,16,37}$ Using a DRS-R98 range on the overall severity score without an a priori assumption about which symptoms would comprise subsyndromal delirium, we found that subsyndromal delirium is distinguished from full syndromal delirium by many symptoms being less severe, specifically motor activity disturbances (retardation, agitation), psychotic symptoms (delusions, hallucinations), higher level thinking (thought process) and cognition (attention, orientation, short-term and long-term memory).
This study utilised a delirium symptom rating scale severity score to define subsyndromal delirium and indicates that a range of delirium symptoms (rather than just the presence of one or two core diagnostic features) are relevant to the concept of subsyndromal illness. Subsyndromal delirium as defined herein may simply reflect milder episodes of delirium. The breadth of symptoms rated on the DRS-R98 is broader than that for DSM-IV and boxplots distributions in the validation study included some milder and resolving cases that met DSM-IV criteria but fell below the best receiver operating characteristics analysis recommended cut-off for full syndromal delirium on the DRS-R98. ${ }^{23}$ This is consistent with the DSM-IV criteria being the most inclusive of delirium as compared with DSM-III-R and ICD classification systems ${ }^{18}$ and therefore probably include mild and subsyndromal delirium as 'delirious'. However, this approach risks lower specificity and opportunity to differentiate outcomes for subsyndromal delirium. Our data suggest that if subsyndromal delirium is of interest, the DRS-R98 cut-off score can be lowered towards eight points so as not to miss individuals with milder or subsyndromal delirium, although at a risk of including some false-positives.

\section{Delirium phenomenology}

Recent work has conceptualised delirium phenomenology as consisting of three core domains: (a) cognitive with disproportionate impairment of attention; (b) circadian disturbance characterised by sleep-wake cycle and motor alterations; and (c) disturbances of higher level thinking (comprehension, language and thinking processes). ${ }^{2,7,38}$ Our longitudinal work supports and complements cross-sectional findings where more prolonged episodes are characterised by more disturbed cognitive and higher-order thinking late in the episode with relatively stable circadian rhythm disturbance. These findings may reflect differences in underlying neurobiology and may have therapeutic implications as to how delirium is best managed as it becomes more persistent.

The extent to which the phenomenological profile in delirium is impacted on by medication exposure is an important and 
poorly addressed issue in delirium research. Our study addresses this issue in more detail than previous work by accounting for exposure to a variety of psychotropic agent classes (and doses) over longitudinal assessments using GEE. A key consideration, for example, is whether subsyndromal delirium reflects delirium that is attenuated by use of medications. We found very limited influence of medications on syndromal status other than a relationship involving benzodiazepines that indicated longer duration and cognitive severity associated with greater use of benzodiazepines. This is consistent with the well-documented finding that benzodiazepine use is an aggravating factor for delirium $^{39}$ and that usual good clinical practice involves reducing/discontinuing benzodiazepine use in individuals with delirium, which is not related to substance withdrawal or seizures. When a measure for non-cognitive features was also incorporated (i.e. DRS-R98 analyses), full syndromal delirium status was predicted by altered motor activity and psychosis rather than benzodiazepine exposure, suggesting that when these features are not included in analyses, elements that relate to their management or aetiology emerge as proxies. This finding emphasises the value of using an assessment method that includes a wide range of cognitive and non-cognitive features in studies of delirium phenomenology.

\section{Shortcomings}

The use of a palliative care population confers particular characteristics (such as high frequency of terminal delirium, more hypoactivity) that may not generalise to other settings so these findings require confirmation in other medical settings and other age groups that have a higher proportion of participants who are not elderly people. However, active screening for delirium usually detects more individuals with hypoactive delirium, whereas referral samples include more individuals with hyperactive delirium because of detection bias. The prevailing concept of delirium is as a unitary syndrome where a relatively consistent clinical picture occurs despite the diversity in aetiology, treatment exposure and vulnerability of population studied. It represents an alteration of normal consciousness in an otherwise aroused cerebral cortex. Traditionally, this concept is based on both decades of descriptive work and clinical experience across medical, surgical and traumatic delirious populations. Also, reports across different medical and surgical populations report the same constellation of symptoms when using the same measurement tools. Recent cross-sectional work by our group has indicated considerable similarity in clinical profile of delirium occurring in individuals screened from palliative care, ${ }^{6}$ an elderly medical referral sample ${ }^{40}$ and a relatively younger, dementia-free referral sample from a consultation-liaison psychiatry service in Northern India. ${ }^{38}$ However, the specific characteristics of people in palliative care do influence delirium profile in some aspects with, for example, a more prominent hypoactive profile of motor activity. ${ }^{41}$

The definition of subsyndromal delirium applied herein (using DRS-R98 score cut-off) identified a subset of participants diagnosed with full syndromal delirium (according to DSM-IV criteria) as having delirium of subsyndromal severity, thus highlighting the need for further research to better understand how to best define subsyndromal delirium. Because we did not include non-delirious controls as a comparison, we cannot highlight which of the subsyndromal delirium symptoms are most differentiating to enhance detection clinically. We followed both individuals with prevalent and incident delirium. Finally, the inclusion of comorbid dementia in a third of individuals makes this cohort representative of real-world populations that develop delirium.

\section{Implications}

This study reports detailed symptom assessment serially over the course of the delirium episodes using highly structured and validated instruments in an adult population that included individuals who were not all elderly people. This work highlights symptoms that are associated with full syndromal delirium and more persistent illness that should therefore be the focus of efforts at detection and diagnosis. Full syndromal delirium is distinguished from subsyndromal delirium by more severe symptoms across most cognitive and non-cognitive symptoms of delirium and more persistent illness is characterised by increasing dominance of cognitive and thinking impairments. David Meagher, MD, PhD, MRCPsych, Department of Adult Psychiatry, Midwestern
Regional Hospital, Limerick, Milford Hospice Palliative Care Centre, Limerick and Regional Hospital, Limerick, Milford Hospice Palliative Care Centre, Limerick and
University of Limerick Medical School, Limerick, Ireland; Dimitrios Adamis, MD, MRCPsych, Research and Academic Institute of Athens, Athens, Greece; Paula Trzepacz, MD, Lilly Research Laboratories, Indianapolis, University of Mississippi Medical School, Jackson, Tufts University School of Medicine, Massachusetts and Indiana University School of Medicine, Indiana, USA; Maeve Leonard, MD, MRCPsych, Department of Adult Psychiatry, Midwestern Regional Hospital, Limerick, Milford Hospice Palliative Care Centre, Limerick and University of Limerick Medical School, Limerick, Ireland

Correspondence: David Meagher, University of Limerick Medical School, Limerick, Ireland. Email: david.meagher@ul.ie

First received 5 Apr 2011, final revision 11 Jul 2011, accepted 15 Sep 2011

\section{References}

1 Siddiqi N, House AO, Holmes JD. Occurrence and outcome of delirium in medical in-patients: a systematic literature review. Age Ageing 2006; 35: 350-64.

2 Trzepacz PT, Meagher DJ, Leonard M. Delirium. In American Psychiatric Publishing Textbook of Psychosomatic Medicine (2nd edn) (ed. J Levenson). American Psychiatric Publishing, 2011.

3 MacLullich AM, Beaglehole A, Hall RJ, Meagher DJ. Delirium and long-term cognitive impairment. Int Rev Psychiatry 2009; 21: 30-42.

4 Meagher D. More attention, less confusion: time to lessen the burden of delirium. Int Rev Psychiatry 2009; 21: 1-3.

5 Meagher DJ, Trzepacz PT. Delirium. In Oxford Textbook of Psychiatry (2nd edn) (eds M Gelder, N Andreasen, J Lopez Ibor Jr, J Geddes): 325-32. Oxford University Press, 2009.

6 Meagher DJ, Moran M, Raju B, Gibbons D, Donnelly S, Saunders J, et al. Phenomenology of delirium. Assessment of 100 adult cases using standardised measures. Br J Psychiatry 2007; 190: 135-41.

7 Franco JG, Trzepacz PT, Mejia MA, Ochoa S. Factor analysis of the Colombian translation of the Delirium Rating Scale (DRS), Revised-98. Psychosomatics 2009; 50: 255-62

8 Gupta N, de Jonghe J, Schieveld J, Leonard M, Meagher D. Delirium phenomenology: what can we learn from the symptoms of delirium? J Psychosom Res 2008; 65: 215-22.

9 Fann JR, Alfano CM, Burington BE, Roth-Roemer S, Katon WJ, Syrjala KL. Clinical presentation of delirium in patients undergoing hematopoietic stem cell transplantation. Cancer 2005; 103: 810-20.

10 Sylvestre MP, McCusker J, Cole M, Regasse A, Belzile E, Abrahamowicz M. Classification of patterns of delirium severity scores over time in an elderly population. Int Psychogeriatrics 2006; 18: 667-80.

11 Rudberg MA, Pompei P, Foreman MD, Ross RE, Cassel CK. The natural history of delirium in older hospitalized patients: a syndrome of heterogeneity. Age Ageing 1997; 26: 169-74.

12 Marcantonio E, Ta T, Duthie E, Resnick NM. Delirium severity and psychomotor types: their relationship with outcomes after hip fracture repair. J Am Geriatr SOC 2002; 50: 850-7.

13 Marcantonio ER, Kiely DK, Simon SE, Salles N, Jenn J, Rasoamanarivo E, et al. Outcomes of older people admitted to postacute facilities with delirium. J Am Geriatr Soc 2005; 53: 963-9.

14 Cole M, McCusker J, Dendukuri N, Han L. The prognostic significance of subsyndromal delirium in elderly medical inpatients. J Am Geriatr Soc 2003; 51: 754-60.

15 Bourdel-Marchasson I, Vincent S, Germain C, Salles N, Jenn J, Rasoamanarivo $\mathrm{E}$, et al. Delirium symptoms and low dietary intake in 
older inpatients are independent predictors of institutionalization: a 1-year prospective population-based study. J Gerontol Biol Med Sci 2004; 59: 350-4.

16 Cole MG, McCusker J, Ciampi A, Belzile E. The 6- and 12-month outcomes of older medical inpatients who recover from subsyndromal delirium. J Am Geriatr Soc 2008; 56: 2093-9.

17 American Psychiatric Association. Diagnostic and Statistical Manual of Mental Disorders (4th edn) (DSM-IV). APA, 1994.

18 Laurila JV, Pitkala $\mathrm{KH}$, Strandberg TE, Tilvis RS. The impact of different diagnostic criteria on prevalence rates for delirium. Dement Geriatr Cogn Dis 2003; 16: 156-62.

19 World Health Organization. The ICD-10 Classification of Mental and Behavioural Disorders: Clinical Descriptions and Diagnostic Guidelines. WHO 1992.

20 American Psychiatric Association. Diagnostic and Statistical Manual of Mental Disorders (3rd edn, revised) (DSM-III-R). APA, 1987.

21 Inouye SK, van Dyck $\mathrm{CH}$, Alessi CA, Balkin S, Siegal AP, Horwitz RI. Clarifying confusion: the confusion assessment method. A new method for detecting delirium. Ann Int Med 1990; 113: 941-8.

22 Ryan $\mathrm{K}$, Leonard $\mathrm{M}$, Guerin S, Donnelly S, Conroy M, Meagher D. Validation of the Confusion Assessment Method in the palliative care setting. Palliative Med 2009; 23: 40-5

23 Trzepacz PT, Mittal D, Torres R, Kanary K, Norton J, Jimerson N. Validation of the Delirium Rating Scale-Revised-98: comparison to the Delirium Rating Scale and Cognitive Test for Delirium. J Neuropsychiatry Clin Neurosci 2001 13: $229-42$.

24 Hart RP, Levenson JL, Sessler CN, Best AM, Schwartz SM, Rutherford LE. Validation of a cognitive test for delirium in medical ICU patients. Psychosomatics 1996; 37: 533-46.

25 Trzepacz PT, Maldonado JR, Kean J, Abell M, Meagher DJ. The Delirium Rating Scale - Revised-98 (DRS-R98) Administration Manual. A Guide to Increase Understanding of How to Solicit Delirium Symptoms to Administer the DRS-R98. December, 2010, Indianapolis, (c) Trzepacz.

26 Kennedy RE, Nakase-Thompson R, Nick TG, Shere M. Use of the cognitive test for delirium in patients with traumatic brain injury. Psychosomatics 2003; 44: 283-9.

27 Hart RP, Best AM, Sessler CN, Levenson JL. Abbreviated Cognitive Test for Delirium. J Psychosomatic Res 1997; 43: 417-23.

28 Royal Pharmaceutical Society. British National Formulary (54th edn). BMJ Publishing Group, 2007.
29 Centorrino P, Eakin M, Bahk WM, Kelleher JP, Goren J, Salvatore P, et al Inpatient antipsychotic drug use in1998, 1993 and1989. Am J Psychiatry 2002; 159: 1932-5.

30 Pereira J, Lawlor P, Vigano A, Dorgan M, Bruera E. Equianalgesic dose ratios for Opioids: a critical review and proposals for long-term dosing. J Pain Symptom Manage 2001; 22: 672-87.

31 World Medical Association. Declaration of Helsinki: Ethical Principles for Medical Research Involving Human Subjects. WMA, 2004 (http://www.wma.net/en/30publications/10policies/b3/).

32 Dolan MM, Hawkes WG, Zimmerman SI, Morrison RS, Gruber-Baldini AL, Hebel JR, et al. Delirium on hospital admission in aged hip fracture patients: prediction of mortality and 2-year functional outcomes. J Gerontology 2000; 55A: M527-34.

33 Gaudreau JD, Gagnon P, Roy MA, Harel F, Tremblay A. Opioid medications and longitudinal risk of delirium in hospitalized cancer patients. Cancer 2007; 109: $2365-73$

34 Cheung CZ, Alibhai SM, Robinson M, Tomlinson G, Chittock D, Drover J, et al. Recognition and labeling of delirium symptoms by intensivists: does it matter? Int Care Med 2008; 34: 437-46.

35 Lipowski ZJ. Transient cognitive disorders (delirium, acute confusional states) in the elderly. Am J Psychiatry 1983; 140: 1426-36.

36 Voyer P, Richard S, Doucet L, Carmichael PH. Detecting delirium and subsyndromal delirium using different diagnostic criteria among demented long-term care residents. J Am Med Dir Assoc 2009; 10: 181-8.

37 Ouimet S, Riker R, Bergeron N, Cossette M, Kavanagh B, Skrobik Y. Subsyndromal delirium in ICU: evidence for a disease spectrum. Int Care Med 2007; 33: 1007-13

38 Grover S, Mattoo S, Chakravarty K, Trzepacz PT, Meagher D, Gupta N. Symptom profile and etiology of delirium in a referral population in Northern India: factor analysis of the DRS-R98. I Neuropsychiatry Clin Neurosci, in press.

39 Clegg A, Young JB. Which medications to avoid in people at risk of delirium: a systematic review. Age Ageing 2011; 40: 23-9.

40 Jabbar F, Leonard M, Meehan K, Cronin C, O'Connor M, Reynolds $\mathrm{P}$, et al. Neuropsychiatric and cognitive profile of patients with DSM-IV delirium referred to an old age psychiatry consultation-liaison service. Int Psychogeriatrics 2011; 23: 1167-74.

41 Meagher D. Motor subtypes of delirium: past, present and future. Int Rev Psychiatry 2009; 21: 59-73. 